\title{
Low- and High-Frequency Insulin Secretion Pulses in Normal Subjects and Pancreas Transplant Recipients: Role of Extrinsic Innervation
}

\author{
Gabriele E. Sonnenberg, Raymond G. Hoffmann, Christopher P. Johnson, * and Ahmed H. Kissebah \\ Division of Endocrinology, Metabolism, and Clinical Nutrition, Department of Medicine, and *Division of Transplant Surgery, \\ Department of Surgery, Medical College of Wisconsin, Milwaukee, Wisconsin 53226
}

\begin{abstract}
Low-frequency ultradian and high-frequency insulin secretion pulses were studied in normal subjects and in metabolically stable pancreas transplant recipients. Insulin secretion pulsatility was evaluated after deconvoluting the pulsatile plasma $\mathbf{C}$ peptide concentrations with its kinetic coefficients. In normal subjects, ultradian insulin secretion pulses with periodicities of 75-115 min were consistently observed during the 24-h secretory cycle. Pulse period and relative amplitude during the overnight rest $(95 \pm 4 \mathrm{~min}$ and $27.6 \pm 2.4 \%)$ were similar to those during the steady state of continuous enteral feeding ( $93 \pm 5 \mathrm{~min}$ and $32.6 \pm 3.3 \%$ ). Sampling at 2 -min intervals revealed the presence of high-frequency insulin secretion pulses with periodicities of 14-20 min and an average amplitude of 46.6 $\pm 5.4 \%$. Pancreas transplant recipients had normal fasting and fed insulin secretion rates. Both low- and high-frequency insulin secretion pulses were present. The high-frequency pulse characteristics were identical to normal. Low-frequency ultradian pulse periodicity was normal but pulse amplitude was increased. Thus, ultradian insulin secretory pulsatility is a consistent feature in normal subjects. The low- and high-frequency secretion pulsatilities are generated independent of extrinsic innervation. Autonomic innervation might modulate low-frequency ultradian pulse amplitude exerting a dampening effect. (J. Clin. Invest. 1992. 90:545-553.) Key words: C peptide $\bullet$ deconvolution $\bullet$ Detect $\bullet$ kinetic-coefficients $\bullet$ pulse characteristics
\end{abstract}

\section{Introduction}

Pancreatic insulin secretion is a highly dynamic process exhibiting temporal variations during the 24 -h secretory cycle. In addition to meal-stimulated responses and circadian rhythmicity, high-frequency pulsations occurring at a periodicity of 9-14 min commonly referred to as oscillations have been observed in humans, rhesus monkeys, baboons, and dogs (1-7). In 24-h sampling protocols with either three meals (8), continuous enteral nutrition (9), or intravenous glucose (10), lower-frequency or ultradian pulses with mean periodicities of 80-120 min have also been reported.

This work was presented in part at the American Federation for Clinical Research Meeting, 1990, Washington, DC.

Address correspondence to Ahmed H. Kissebah, M.D., Ph.D., Division of Endocrinology, Metabolism, and Clinical Nutrition, Froedtert Memorial Lutheran Hospital, 9200 W. Wisconsin Avenue, Milwaukee, WI 53226.

Received for publication 22 May 1991 and in revised form 18 February 1992.

J. Clin. Invest.

(C) The American Society for Clinical Investigation, Inc.

$0021-9738 / 92 / 08 / 0545 / 09 \$ 2.00$

Volume 90, August 1992, 545-553
The potential physiological role of pulsatile insulin secretion has been reviewed recently (11). Analogous to engineering systems, the frequency-encoded regulation of insulin-glucose homeostasis should be more precise and economical when target organ exposure occurs in an interrupted rather than in a continuous mode. Comparing high-frequency pulsatile versus continuous insulin administration, the pulsatile mode results in greater hypoglycemic effects (12), greater suppression of hepatic glucose production (13), more pronounced inhibition of arginine-stimulated glucagon secretion (14), and higher insulin-specific binding to monocytes (12). More recently, it has been suggested that the physiologically occurring mode of pulsatile insulin delivery provides an optimal concentration differential and time frame for the reduction and return of cell surface insulin receptors in perifused rat hepatocytes (15). In all of these studies, the frequency and amplitude of administered insulin simulated the high-frequency oscillatory mode. Whether the low-frequency ultradian pulsatile mode exerts similar or complementary functions is unknown.

That the high-frequency pulsatile insulin secretion is generated within the pancreas is supported by the finding of regular pulsations during the in vitro perfusion of isolated canine pancreata (16). Cholinergic and adrenergic receptor blocking agents added to the perfusion medium do not influence this mode, indicating that its generation is not controlled by classical autonomic innervation. Ganglionic nerve blocking agents, on the other hand, result in shorter periodicity and irregular amplitude pulsations, suggesting the existence of an intrapancreatic ganglionic system serving as a pacemaker and/or an integration center $(17,18)$. More recently, Longo et al. (19) suggested that the high-frequency insulin secretion pulsatility represents an intrinsic feature of the $\beta$ cell, driven by periodic fluctuations in intracellular calcium and metabolic activity. Detailed characteristics of the low-frequency ultradian insulin secretion pulses and their relationship to the $\beta$ cell activity are unclear. Furthermore, generation and regulation of these pulses and, in particular, the role of extrinsic autonomic innervation are unknown.

The present study was undertaken to characterize the ultradian insulin secretion pulsatility during the unstimulated and nutrient-stimulated states and to define the role of extrinsic innervation in the generation and regulation of these pulses. Low-frequency ultradian and high-frequency pulsatile insulin secretion modes were evaluated in healthy normal subjects and in metabolically stable pancreas transplant recipients. The latter should provide an in vivo model of pancreatic denervation.

\section{Methods}

Subjects

Normal subjects. Eight nonobese normal women (body mass index: $20.2-23.4 \mathrm{~kg} / \mathrm{m}^{2}$ ) participated in the study. Their weight was stable for $\geq 2$ mo before the study. Subjects were healthy without evidence sug- 
gestive of diabetes mellitus or other endocrine disease, hypertension, or heart disease. These disorders were excluded by history, physical examination, electrocardiogram, and chest $\mathrm{x}$ ray. Normal values for glucose tolerance, liver, kidney, and thyroid function were ascertained by laboratory studies. Subjects' clinical characteristics are shown in Table I.

Pancreas transplant recipients. Six subjects (four women and two men ) with a history of insulin-dependent diabetes mellitus for 17-29 yr (mean \pm SEM: $22.3 \pm 1.6$ ) were studied 4-10 mo (6.4 \pm 1.3$)$ after pancreas transplantation. They also received kidney transplants. Cadaveric kidney and whole pancreas grafts were implanted in the pelvic region. Exocrine pancreatic secretion was drained directly into the urinary bladder by duodenocystostomy (20). Arterial blood supply was established via an anastomosis between the iliac artery (recipient) and the reconstructed splenic superior mesenteric arteries (donor). Insulin is delivered directly into the systemic circulation via an anastomosis between the external iliac vein (recipient) and the portal vein stump (donor). All extrinsic innervation had been severed during the explantation and procurement procedures.

The transplant recipients were of normal weight (body mass index: $20.2-26.7 \mathrm{~kg} / \mathrm{m}^{2}$; Table I) and had good renal function (serum creatinine $1.45 \pm 0.16 \mathrm{mg} / \mathrm{dl}$ ). At the time of the study, none of the subjects required exogenous insulin supplementation. Subjects were metabolically stable, their fasting and random plasma glucose levels being within the normal range. Subjects continued to take their immunosuppressive medications consisting of Cyclosporine A (range 400-500 mg/ d), azathioprine (range $50-100 \mathrm{mg} / \mathrm{d}$ ), and prednisone (range 15-20 $\mathrm{mg} / \mathrm{d}$ ).

Studies were performed at the Clinical Research Center of the Medical College of Wisconsin. The study protocol was approved by the Institutional Human Research Review Committee. Written informed consent was obtained from each subject before investigation.

\section{Experimental procedures}

Evaluation of low- and high-frequency pulses in plasma C-peptide lev$e l s$. In the normal subjects, low-frequency ultradian insulin secretion pulse characteristics were evaluated during two phases: $12 \mathrm{~h}$ of continu- ous enteral nutrition and $12 \mathrm{~h}$ of overnight rest. Continuous enteral nutrition was chosen to provide a constant pancreatic stimulus, thus avoiding the large secretory increments accompanying oral feeding and the potential impediment of these on ultradian pulse differentiation and detection. Studies in normal subjects demonstrated consistent periodicity and pulse characteristics between the overnight and nutrientstimulated phases (see Results). Furthermore, after the initial response to feeding, the insulin secretion profile reached an apparent steady state during the last $8 \mathrm{~h}$. To reduce the total amount of blood withdrawn in the transplant recipients, ultradian pulse evaluation was limited to the last $8 \mathrm{~h}$ of the enteral feeding period. Blood samples were continuously removed over $10-$ min periods $(1 / 6$ to $1 / 12$ the anticipated periodicity) using an automated withdrawal system.

A feeding tube (Frederick Miller Feeding Tube Set ${ }^{R}$, Cook, Bloomington, IN) was placed into the lower third of the duodenum under fluoroscopic control. A liquid formula (TwoCal $\mathrm{HN}^{\mathrm{R}}$; Ross Laboratories, Columbus, $\mathrm{OH}$, containing $17 \%$ protein, $40 \%$ fat, and $43 \%$ carbohydrates) was administered at a rate of $150 \mathrm{kcal} / \mathrm{h}$. Nutrient flow was maintained constant using a feeding pump (Kangoroo ${ }^{R}$ 324; Sherwood Medical Industries Inc., St. Louis, MO). Enteral feeding was started at 9:00 a.m. and continued until 9:00 p.m.

Blood samples were drawn from a peripheral arm vein using a thrombo-resistant needle and tubing set (Kowarski-Dakmed 25200; Dakmed Inc., Buffalo, NY) connected to a peristaltic continuous withdrawal pump (Dakmed Ambulatory Withdrawal Pump, model ML6$5 \mathrm{H}$; Dakmed Inc.). The pump speed was adjusted to obtain blood volumes of $2.2 \mathrm{ml}$ over each 10 -min interval. To prevent clotting at the tube end, heparin $(10,000 \mathrm{U} / \mathrm{ml})$ was infused into the proximal $6 \mathrm{~cm}$ of the tubing at a rate of $1 \mu \mathrm{l} / \mathrm{min}$ using a syringe-driven pump (Betatron II, model 9200; CPI/Lilly, St. Paul, MN). Blood was collected in heparinized tubes placed in a fraction collector (Retriever $\mathbf{I I}^{\mathbf{R}}$, Isco, Lincoln, NE). Blood samples were kept on ice until centrifuged, and plasma was stored at $-70^{\circ} \mathrm{C}$ until assayed. Samples were analyzed for determination of plasma C-peptide. Another aliquot was analyzed for insulin and glucose levels. Pilot studies have demonstrated that the amount of heparin delivered into the sample had no significant effect on the radioimmunoassayable hormone levels.

Table I. Clinical Characteristics of the Study Groups

\begin{tabular}{|c|c|c|c|c|c|c|c|}
\hline \multirow[b]{2}{*}{ Subject } & \multirow[b]{2}{*}{ Age } & \multirow[b]{2}{*}{ Weight } & \multirow[b]{2}{*}{ BMI } & \multicolumn{2}{|c|}{ Glucose } & \multicolumn{2}{|c|}{ Insulin } \\
\hline & & & & Fasting & Fed & Fasting & Fed \\
\hline & $y r$ & $k g$ & $\mathrm{~kg} / \mathrm{m}^{2}$ & \multicolumn{2}{|c|}{$m g / d l$} & \multicolumn{2}{|c|}{$\mu U / m l$} \\
\hline \multicolumn{8}{|l|}{ Normal subjects } \\
\hline 1 & 27 & 60 & 23.4 & 78 & 87 & 9.1 & 12.4 \\
\hline 2 & 41 & 57 & 23.1 & 86 & 103 & 3.7 & 16.7 \\
\hline 3 & 34 & 54 & 20.3 & 81 & 97 & 10.5 & 18.0 \\
\hline 4 & 38 & 58 & 21.6 & 84 & 99 & 8.2 & 22.4 \\
\hline 5 & 28 & 57 & 20.2 & 81 & 97 & 4.3 & 16.6 \\
\hline 6 & 23 & 55 & 21.8 & 79 & 103 & 5.0 & 16.9 \\
\hline 7 & 26 & 61 & 21.6 & 81 & 93 & 5.3 & 15.7 \\
\hline 8 & 28 & 65 & 21.0 & 82 & 90 & 9.0 & 15.2 \\
\hline Mean \pm SEM & $31 \pm 2.2$ & $58 \pm 1.3$ & $21.6 \pm 0.4$ & $81.5 \pm 0.9$ & $96.1 \pm 2.1$ & $6.9 \pm 1.0$ & $16.8 \pm 1.0$ \\
\hline \multicolumn{8}{|c|}{ Pancreas transplant recipients } \\
\hline 1 & 26 & 60 & 26.7 & 71 & 79 & 28 & 89 \\
\hline 2 & 31 & 69 & 23.6 & 93 & 118 & 23 & 65 \\
\hline 3 & 36 & 54 & 21.9 & 99 & 112 & 13 & 32 \\
\hline 4 & 34 & 56 & 23.4 & 86 & 102 & 35 & 110 \\
\hline 5 & 43 & 56 & 20.2 & 84 & 95 & 25 & 63 \\
\hline 6 & 33 & 73 & 25.5 & 77 & 156 & 17 & 72 \\
\hline Mean \pm SEM & $34 \pm 2.3$ & $61 \pm 3.2$ & $23.6 \pm 1.0$ & $85.0 \pm 4.2$ & $110.3 \pm 10.7$ & $23.5 \pm 3.2^{*}$ & $71.8 \pm 10.7^{*}$ \\
\hline
\end{tabular}

* Significantly different from normal subjects, $P<0.001$ or less. BMI, body mass index. 
High-frequency insulin secretion pulsations were evaluated during a 90-min period after an overnight fast. Blood sampling was performed over 2-min intervals ( $1 / \mathrm{s}$ to $1 / 10$ the anticipated periodicity).

C-peptide kinetic coefficients. Kinetic coefficients of C-peptide turnover were determined in each individual as described previously (21, 22). Calculation of insulin secretion rate was based upon the rationale that insulin and C-peptide are secreted from the pancreas in equimolar amounts and that C-peptide, unlike insulin, is not significantly extracted by the liver. Thus, pancreatic insulin secretion can be estimated by deconvoluting the plasma $\mathrm{C}$-peptide concentrations using the kinetic coefficients of $\mathrm{C}$-peptide in each individual.

After an overnight fast, an intravenous catheter was inserted into an antecubital vein for the administration of a C-peptide bolus. A forearm vein of the contralateral arm was retrogradely cannulated by an intravenous catheter for blood sampling. The forearm was placed in a warming chamber kept at $70^{\circ} \mathrm{C}$. Basal C-peptide levels were determined in arterialized venous blood samples obtained at 10 -min intervals over a 30-min period. An intravenous bolus containing $50 \mathrm{nmol}$ Biosynthetic Human C-Peptide (kindly provided by Eli Lilly and Company, Lilly Research Laboratories, Indianapolis, IN) diluted in saline was administered. Blood samples were taken at 2-4-min intervals during the first hour and at 10-20-min intervals during the second hour.

\section{Analytical procedures}

Radioimmunoassay of plasma C-peptide was performed using a specific antibody to human C-peptide (Incstar Corp., Stillwater, NM). All samples were measured in triplicate. To assess the stability and reliability of the radioimmunoassay, quality controls were performed as suggested by other studies of hormone pulsatility (23). Seven pool sera of increasing C-peptide concentrations were used to evaluate the intraand interassay coefficients of variation $(\mathrm{CV}) .{ }^{1}$ Variance was calculated according to Rodbard (24) using the program CLR ANOVA (Clear Lakes Research, Houston, TX). The intraassay CV ranged between 2.8 and $5.4 \%$ and the interassay $\mathrm{CV}$ between 5.9 and $9.5 \%$ over a concentration range of $1.6-8.2 \mathrm{ng} / \mathrm{ml}$. A solid-phase radioimmunoassay (Coat-a-Count Insulin; Diagnostic Products Corp., Los Angeles, CA) was used for the measurement of plasma insulin. Plasma glucose concentrations were measured by the glucose oxidase method using a Glucose Analyzer 2 (Beckman Instruments Inc., Brea, CA).

\section{Calculations and data analyses}

$C$-peptide turnover kinetic coefficients and insulin secretion rate. The plasma disappearance curve of C-peptide after the Biosynthetic $\mathrm{Hu}$ man C-Peptide bolus injection was analyzed using a mathematical twocompartment model representing the plasma and the extravascular pool(s). $K_{01}$ is the fractional removal rate and $K_{12}$ and $K_{21}$ are the equilibration constants between the two compartments. Compartmental analysis was performed using the SAAM program (25). In normal subjects, mean C-peptide kinetic coefficients were as follows: $K_{01}$ : $0.0471 \pm 0.0019, K_{12}: 0.0324 \pm 0.0053$, and $K_{21}: 0.0359 \pm 0.006 \mathrm{~min}^{-1}$. In the kidney/pancreas transplant recipients, the fractional removal rate $K_{01}$ was reduced to $0.0221 \pm 0.0015$, and $K_{12}$ and $K_{21}$ averaged $0.0397 \pm 0.0050$ and $0.0680 \pm 0.0131 \mathrm{~min}^{-1}$.

The integro-differential equations used to deconvolute plasma $\mathrm{C}$ peptide were those described by Eaton and co-workers (26). Conversion of C-peptide to insulin equivalents was based upon a mol wt of 3,300 for C-peptide and 6,000 for insulin and a relationship of $40 \mathrm{ng}$ for each milliunit of insulin. Plasma volume was calculated as $4.1 \%$ of ideal body weight $\pm 1 \%$ of subject's actual body weight.

Pulse detection and characterization, variance analysis. Before pulse detection, random fluctuation in triplicate C-peptide measurements due to the radioimmunoassay was determined using the program Predetect (27). Considering five different variance models, the best model characterized by the smallest mean square error was entered into the peak detection program Detect $(27,28)$. The presence of low

1. Abbreviation used in this paper: $\mathrm{CV}$, coefficient of variation. frequency ultradian and high frequency pulses was first tested by examining the plasma C-peptide concentration data using the same program. This action was necessary to exclude false pulsatile patterns created by the deconvolution process itself. Data were also evaluated statistically to ensure that differences between peaks and nadirs exceeded three times the standard error of the triplicate C-peptide measurements at this level. In pilot studies, the efficacy of the Detect versus Cluster (29) program was compared, and a close agreement in pulse detection and periodicity was observed.

The peak detection module of Detect identifies the beginning and end of each pulse by testing the slope of the curve over four, three, and two points and comparing these with successively more stringent criteria to determine whether the curve is increasing, decreasing, or remaining at the same level. The criteria for identifying a significant rise or fall include the variance model after adjusting for the effect of deconvolution. Based on the variance at each point, a threshold is set to separate the signal (the pulse) from the noise (random variation in the means). To further reduce the variability, smoothing of the data is performed by a 1-2-1 polynomial smoother ( $1 / 4$ the earlier value plus $1 / 2$ the current value plus $1 / 4$ the next value). Compared with the $C$-peptide analyses, the deconvolution and smoothing processes did not influence the de-
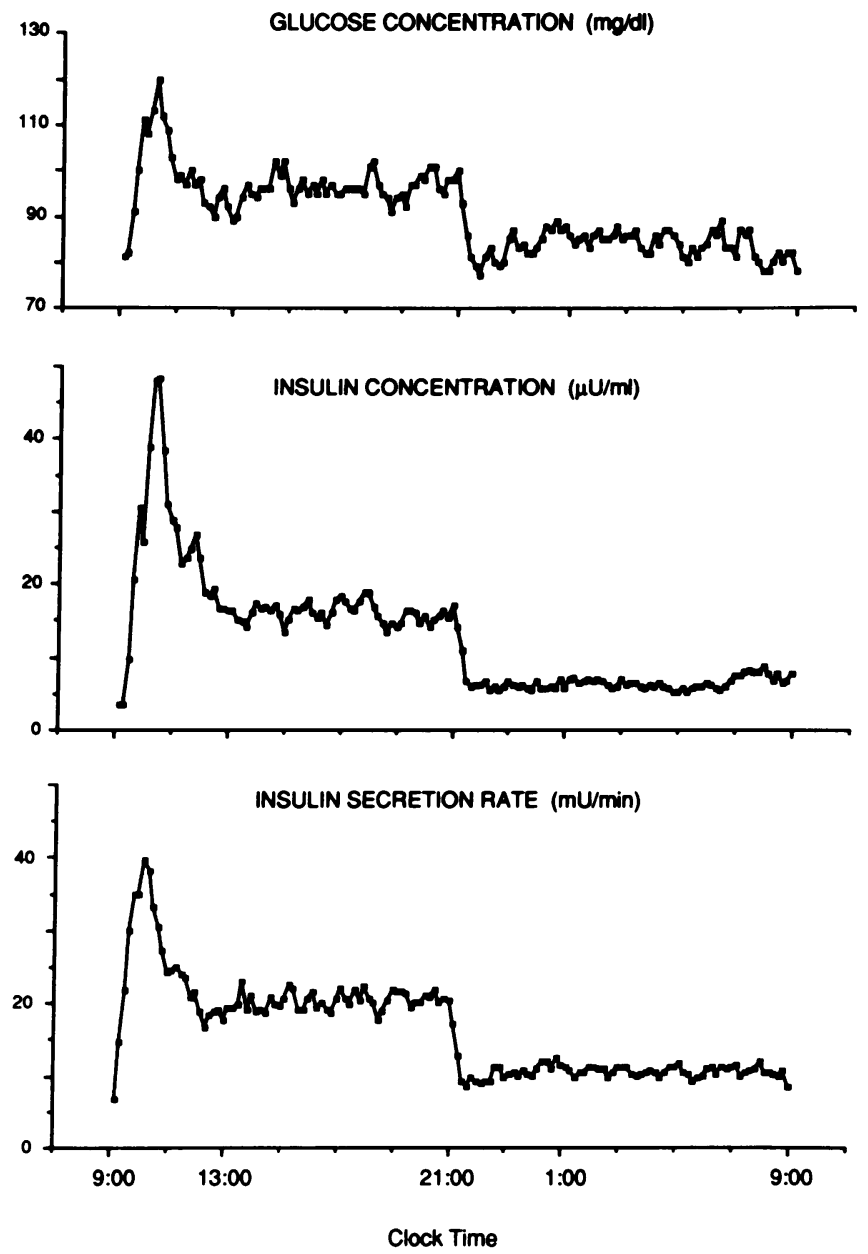

Figure 1. Mean plasma glucose and insulin concentrations and mean insulin secretion rate during nutrient stimulation $(12 \mathrm{~h})$ and overnight rest $(12 \mathrm{~h})$ in normal subjects. Blood samples were continuously withdrawn over 10-min periods using an automated withdrawal system. Results are means obtained from eight subjects. 

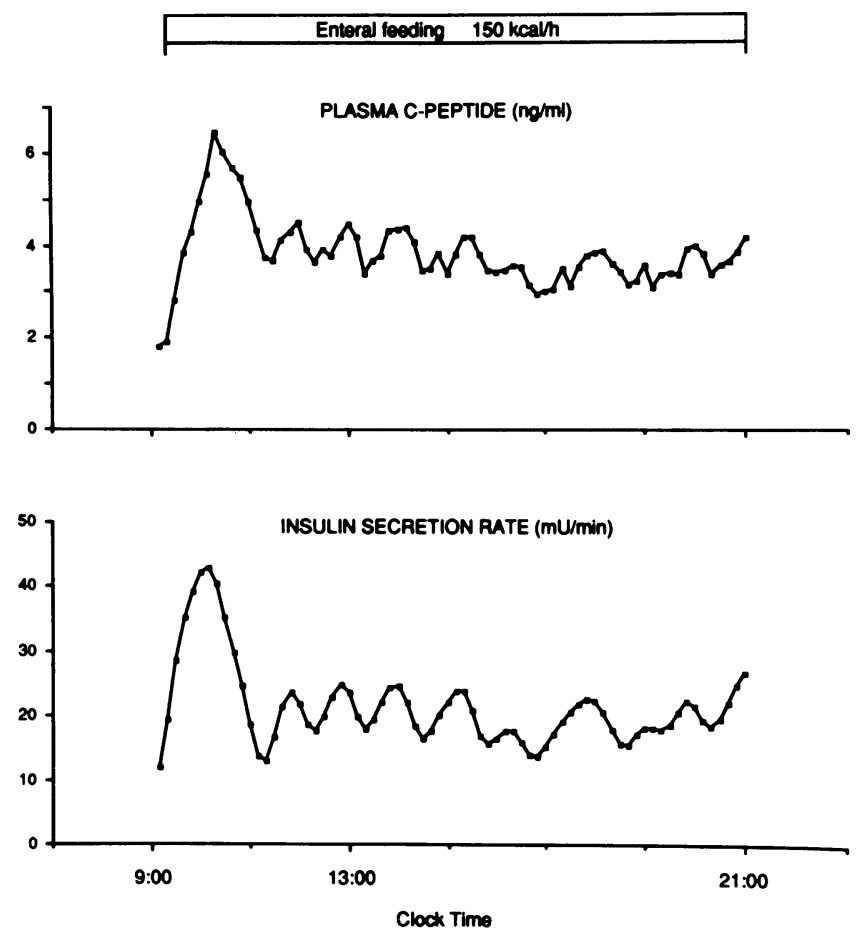

Figure 2. Low-frequency ultradian pulsatility of plasma C-peptide and insulin secretion rate during continuous enteral feeding in a normal subject. C-peptide concentrations were deconvoluted using the individual C-peptide coefficients to calculate the insulin secretion rate. Data were subjected to polynomial smoothing before pulse detection.

tection and periodicity of the pulsatile patterns. Identical procedures were used to compare the pancreas transplant recipients to control subjects.

Pulses were characterized in terms of duration (width of each individual pulse), interval (width between pulses), and period (duration plus prepulse interval), pulse maximum (highest value within the pulse), nadir (lowest value before pulse increment), absolute amplitude (difference between maximal height and nadir), and relative amplitude (percent maximum increase above nadir). In each individual data set, the $\mathrm{CV}$ for pulse period, maximum, and nadir was calculated.

Data are presented as mean \pm SEM. Paired and unpaired $t$ tests were used for data comparison within and between subject groups, respectively.

\section{Results}

Low- and high-frequency insulin secretion pulse characteristics in normal subjects. In normal subjects, insulin secretion averaged $23.7 \pm 1.7 \mathrm{U} / 24 \mathrm{~h}$ of which $15.8 \pm 1.0 \mathrm{U}$ was released during $12 \mathrm{~h}$ of enteral feeding and $7.9 \pm 0.8 \mathrm{U}$ during the remaining $12 \mathrm{~h}$ of overnight rest. During these two phases, peripheral insulin averaged $18.9 \pm 1.3$ and $6.5 \pm 1.0 \mu \mathrm{U} / \mathrm{ml}$ and glucose $97.1 \pm 1.8$ and $83.5 \pm 1.3 \mathrm{mg} / \mathrm{dl}$. Initiation of enteral feeding resulted in a pronounced initial secretory response reaching a peak of $41.9 \pm 4.1 \mathrm{mU} / \mathrm{min}$ after $59 \pm 5 \mathrm{~min}$ and a nadir of $15.0 \pm 1.4 \mathrm{mU} / \mathrm{min}$ after $140 \pm 13 \mathrm{~min}$. After this initial response, an apparent steady state in insulin secretion was observed. During the last $8 \mathrm{~h}$ of the feeding protocol, insulin secretion rate averaged $1.25 \pm 0.09 \mathrm{U} / \mathrm{h}$, and plasma insulin and glucose concentrations averaged $16.8 \pm 1.0 \mu \mathrm{U} / \mathrm{ml}$ and $96.1 \pm 2.1 \mathrm{mg} / \mathrm{dl}$, respectively. During the last $8 \mathrm{~h}$ of the over- night steady state, insulin secretion rate decreased to approximately half and averaged $0.65 \pm 0.07 \mathrm{U} / \mathrm{h}$. The corresponding plasma insulin and glucose concentrations were $6.5 \pm 1.0 \mu \mathrm{U} /$ $\mathrm{ml}$ and $83.3 \pm 1.3 \mathrm{mg} / \mathrm{dl}$. Fig. 1 shows the time course of insulin secretion rate and corresponding peripheral plasma insulin and glucose concentrations during the $24-\mathrm{h}$ study period.

Figs. 2 and 3 show examples of the time course changes in plasma C-peptide concentrations and the corresponding profiles in insulin secretion rates. Although ultradian pulses could have been detected earlier, only the steady state 8-h periods of feeding and overnight rest were subjected to pulse analysis. Thus, a 4-h equilibration period was allowed to avoid potential masking effects of feeding initiation and cessation on pulse characteristics.

In all subjects, low-frequency ultradian insulin secretion pulses were observed during both the nutrient-stimulated and unstimulated states. Pulses appeared to be broad with short intervals between increments and decrements, suggesting a monophasic rather than a biphasic cycle pattern. During continuous enteral feeding, mean pulse duration, interval, and period were $81 \pm 5,11 \pm 1$, and $93 \pm 5 \mathrm{~min}$, respectively (Table II). Individual pulse periods ranged between 75 and $115 \mathrm{~min}$. Mean pulse maximum, nadir, and amplitude were $24.4 \pm 1.5$, $16.5 \pm 1.5$, and $7.9 \pm 0.8 \mathrm{mU} / \mathrm{min}$, respectively. The relative pulse amplitude represented $32.6 \pm 3.3 \%$ of maximum pulse height. During the steady state of overnight rest, ultradian insulin secretion pulses were also observed. The mean duration, interval, and period were $85 \pm 4,10 \pm 0$, and $95 \pm 4 \mathrm{~min}$, respectively, and did not differ from those values observed during continuous enteral feeding. The twofold lower insulin secretion rate was well reflected by twofold lower pulse maximum, nadir, and amplitude $(12.3 \pm 1.2,8.9 \pm 1.1$, and $3.4 \pm 0.4 \mathrm{mU} /$ min, respectively). Thus, the relative pulse amplitude aver-
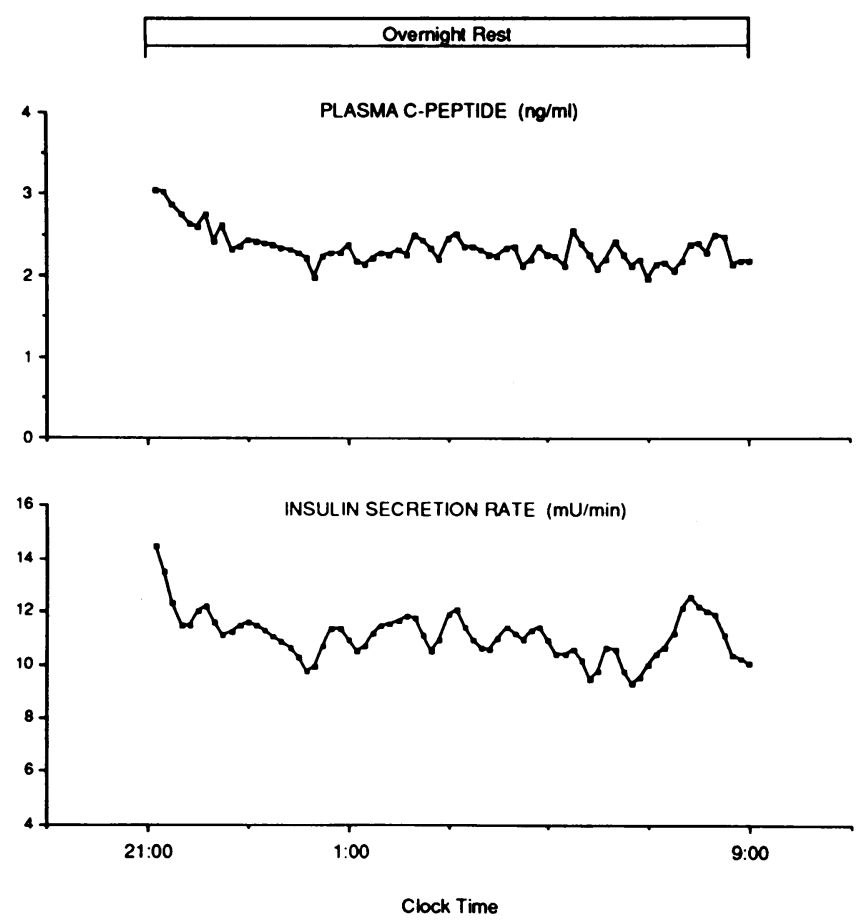

Figure 3. Low-frequency ultradian pulsatility of plasma C-peptide and insulin secretion rate during overnight rest in a normal subject. Data were deconvoluted and smoothed as described in Fig. 2. 
Table II. Ultradian Insulin Secretion Pulse Characteristics in Normal Subjects

\begin{tabular}{|c|c|c|c|c|c|c|c|c|}
\hline Subject & Duration & Interval & Period & Maximum & Nadir & Amplitude & $\begin{array}{c}\text { Relative } \\
\text { amplitude }\end{array}$ & ISR \\
\hline & & $\min$ & & & $\mathrm{mU} / \mathrm{min}$ & & $\%$ & $U / h$ \\
\hline \multicolumn{9}{|c|}{$\begin{array}{l}\text { Continuous enteral nutrition } \\
\text { (8-h steady state) }\end{array}$} \\
\hline 1 & 95 & 15 & 110 & 21.1 & 10.2 & 10.9 & 52 & 0.94 \\
\hline 2 & 105 & 10 & 115 & 30.2 & 19.0 & 11.2 & 37 & 1.57 \\
\hline 3 & 65 & 10 & 75 & 20.5 & 14.9 & 5.6 & 27 & 1.15 \\
\hline 4 & 68 & 10 & 78 & 24.0 & 17.8 & 6.2 & 26 & 1.23 \\
\hline 5 & 74 & 10 & 84 & 23.5 & 14.8 & 8.7 & 37 & 1.16 \\
\hline 6 & 76 & 10 & 86 & 18.6 & 12.5 & 6.1 & 33 & 0.90 \\
\hline 7 & 88 & 10 & 98 & 28.5 & 21.4 & 7.1 & 25 & 1.50 \\
\hline 8 & 80 & 14 & 94 & 28.4 & 21.4 & 7.0 & 24 & 1.53 \\
\hline Mean \pm SEM & $81 \pm 5$ & $11 \pm 1$ & $93 \pm 5$ & $24.4 \pm 1.5^{*}$ & $16.5 \pm 1.5^{*}$ & $7.9 \pm 0.8^{*}$ & $32.6 \pm 3.3$ & $1.25 \pm 0.09^{*}$ \\
\hline $\begin{array}{l}\text { Overnight rest } \\
\text { (8-h steady state }\end{array}$ & & & & $\cdot$ & & & & \\
\hline 1 & 76 & 10 & 86 & 8.2 & 6.1 & 2.1 & 26 & 0.44 \\
\hline 2 & 80 & 10 & 90 & 13.6 & 9.7 & 4.0 & 29 & 0.70 \\
\hline 3 & 95 & 10 & 105 & 12.4 & 7.2 & 5.2 & 42 & 0.62 \\
\hline 4 & 90 & 10 & 100 & 12.1 & 9.1 & 3.0 & 25 & 0.65 \\
\hline 5 & 80 & 10 & 90 & 11.3 & 8.9 & 2.4 & 21 & 0.60 \\
\hline 6 & 78 & 10 & 88 & 7.5 & 5.2 & 2.3 & 31 & 0.40 \\
\hline 7 & 107 & 10 & 117 & 17.9 & 13.5 & 4.4 & 25 & 0.96 \\
\hline 8 & 72 & 10 & 82 & 15.2 & 11.8 & 3.4 & 22 & 0.82 \\
\hline Mean \pm SEM & $85 \pm 4$ & $10 \pm 0$ & $95 \pm 4$ & $12.3 \pm 1.2$ & $8.9 \pm 1.1$ & $3.4 \pm 0.4$ & $27.6 \pm 2.4$ & $0.65 \pm 0.07$ \\
\hline
\end{tabular}

* Significantly higher than overnight rest period, $P<0.001$ or less.

aged $27.6 \pm 2.4 \%$, which is similar to that observed during the fed state.

In the fasting state during the 90 -min sampling period, insulin secretion averaged $0.61 \pm 0.06 \mathrm{U} / \mathrm{h}$. Plasma insulin and glucose concentrations averaged $6.9 \pm 1.0 \mathrm{mU} / \mathrm{ml}$ and $81.5 \pm 0.9 \mathrm{mg} / \mathrm{dl}$, respectively. Sampling at 2-min intervals revealed high-frequency pulsations with short interpulse intervals. The mean duration, interval, and period were $14.6 \pm 0.7$,

Table III. Oscillatory Insulin Secretion Pulses (Fasting State)

\begin{tabular}{|c|c|c|c|c|c|c|c|c|}
\hline Subject & Duration & Interval & Period & Maximum & Nadir & Amplitude & $\begin{array}{c}\text { Relative } \\
\text { amplitude }\end{array}$ & ISR \\
\hline & & $\min$ & & & $\mathrm{mU} / \mathrm{min}$ & & $\%$ & $U / h$ \\
\hline \multicolumn{9}{|l|}{ Normal subjects } \\
\hline 1 & 11.4 & 2.0 & 13.6 & 15.4 & 4.1 & 11.3 & 73 & 0.58 \\
\hline 2 & 15.0 & 2.0 & 17.0 & 14.0 & 7.4 & 6.6 & 47 & 0.65 \\
\hline 3 & 12.8 & 2.0 & 14.8 & 7.0 & 3.2 & 3.8 & 55 & 0.33 \\
\hline 4 & 17.6 & 2.0 & 19.6 & 13.0 & 7.6 & 5.4 & 41 & 0.63 \\
\hline 5 & 14.0 & 1.6 & 15.6 & 9.9 & 6.7 & 3.2 & 32 & 0.75 \\
\hline 6 & 16.0 & 2.0 & 18.0 & 10.2 & 7.2 & 3.0 & 29 & 0.52 \\
\hline 7 & 16.0 & 2.0 & 18.0 & 17.6 & 7.0 & 10.6 & 60 & 0.51 \\
\hline 8 & 13.6 & 2.0 & 15.6 & 18.6 & 11.9 & 6.6 & 36 & 0.91 \\
\hline Mean \pm SEM & $14.6 \pm 0.7$ & $2.0 \pm 0.05$ & $16.6 \pm 0.7$ & $13.2 \pm 1.4$ & $6.9 \pm 0.9$ & $6.3 \pm 1.1$ & $46.6 \pm 5.4$ & $0.61 \pm 0.06$ \\
\hline \multicolumn{9}{|c|}{ Pancreas transplant recipients } \\
\hline 1 & 15.6 & 2.0 & 17.6 & 16.6 & 6.9 & 9.7 & 58 & 0.72 \\
\hline 2 & 17.0 & 3.0 & 20.0 & 18.0 & 5.4 & 12.6 & 70 & 0.65 \\
\hline 3 & 14.4 & 2.4 & 16.8 & 7.9 & 4.4 & 3.5 & 44 & 0.35 \\
\hline 4 & 10.6 & 2.0 & 12.6 & 11.0 & 4.8 & 6.2 & 56 & 0.56 \\
\hline 5 & 14.0 & 2.0 & 16.0 & 14.5 & 6.7 & 7.8 & 54 & 0.57 \\
\hline 6 & 11.2 & 3.6 & 14.8 & 10.7 &, 5.0 & 5.7 & 53 & 0.48 \\
\hline Mean \pm SEM & $13.8 \pm 1.0$ & $2.5 \pm 0.3$ & $16.3 \pm 1.0$ & $13.1 \pm 1.6$ & $5.5 \pm 0.4$ & $7.6 \pm 1.3$ & $55.8 \pm 3.5$ & $0.56 \pm 0.05$ \\
\hline
\end{tabular}

No significant differences between study groups. 


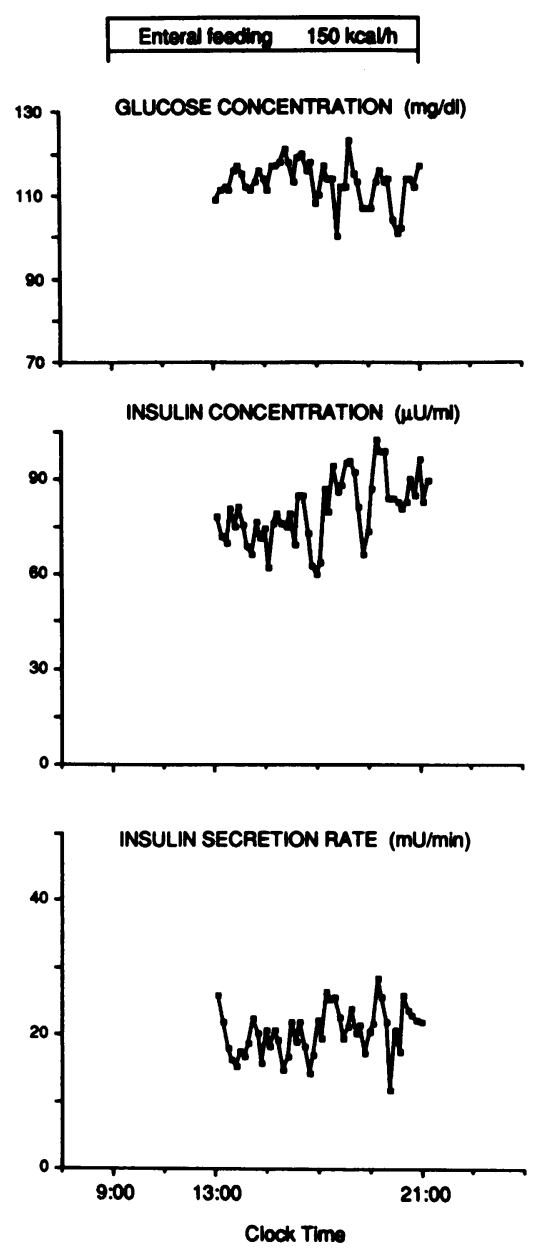

Figure 4. Mean plasma glucose and insulin concentrations and mean insulin secretion rate during the steady state $(8 \mathrm{~h})$ of continuous enteral feeding in pancreas transplant recipients. Blood samples were continuously withdrawn over 10 -min periods using an automated withdrawal system. Results are means obtained from six subjects.

$2.0 \pm 0.05$, and $16.6 \pm 0.7 \mathrm{~min}$, respectively, with individual periods ranging between 13.6 and $19.6 \mathrm{~min}$ (Table III). Mean pulse maximum, nadir, and amplitude were 13.2 \pm 1.4 , $6.9 \pm 0.9$, and $6.3 \pm 1.1 \mathrm{mU} / \mathrm{min}$, respectively. The relative pulse amplitude averaged $46.6 \pm 5.4 \%$ of maximum pulse height.

Low- and high-frequency insulin secretion pulse characteristics in pancreas transplant recipients: influence of extrinsic innervation. The pancreas transplant recipients were metabolically stable. The mean insulin secretion rate during the 8-h steady state of feeding was similar to normal ( $1.25 \pm 0.11$ vs. $1.25 \pm 0.09 \mathrm{U} / \mathrm{h} ; P>0.05)$. Mean plasma glucose was slightly higher $(110 \pm 11$ vs. $96.1 \pm 2.1 \mathrm{mg} / \mathrm{dl} ; P>0.05)$ whereas peripheral plasma insulin level was increased by approximately fourfold ( $71.8 \pm 10.7$ vs. $16.8 \pm 1.0 \mu \mathrm{U} / \mathrm{ml} ; P<0.001)$. These characteristics are shown in Fig. 4.

Transplanted subjects exhibited strong low-frequency ultradian pulse patterns with periodicity parameters similar to normal. Mean pulse duration, interval, and period were $75.8 \pm 5.8,10.5 \pm 0.5$, and $86.3 \pm 6.1 \mathrm{~min}$, respectively. Individual periods ranged between 64 and $106 \mathrm{~min}$ (Table IV). The $\mathrm{CV}$ in pulse periods averaged $25 \pm 4 \%$ and was not significantly different from that found in normal subjects $(20 \pm 2 \% ; P$ $>0.05)$. However, the pulse maxima and nadirs exhibited more pronounced excursions, resulting in significantly greater amplitudes ( $16.1 \pm 3.6$ vs. $7.9 \pm 0.8 \mathrm{mU} / \mathrm{min} ; P=0.023)$. The mean relative pulse amplitude was also increased ( $53 \pm 5$ vs. $33 \pm 3 \% ; P=0.004$ ). The higher maxima and lower nadirs exhibited significantly larger variability from pulse to pulse. The $\mathrm{CV}$ for the maximum height was $16.6 \pm 2.0 \%$ (normal: $9.3 \pm 1.7 \% ; P=0.022$ ) and for the nadir was $35.9 \pm 8.7 \%$ (normal: $12.2 \pm 2.3 \% ; P=0.017$ ). Fig. 5 shows results obtained from a pancreas transplant recipient.

During the 90 -min sampling period in the fasting state, the mean insulin secretion rate was $0.56 \pm 0.04 \mathrm{U} / \mathrm{h}$, which is similar to normal $(0.61 \pm 0.06 \mathrm{U} / \mathrm{h} ; P>0.05)$. Plasma glucose was also normal ( $85 \pm 4$ vs. $82 \pm 1 \mathrm{mg} / \mathrm{dl} ; P>0.05$ ) whereas peripheral insulin concentration was threefold higher (23.5 \pm 3.2 vs. $6.9 \pm 1.0 \mu \mathrm{U} / \mathrm{ml} ; P<0.001)$. High-frequency secretion pulsatility was present in all transplanted subjects with periodicity parameters identical to normal (Table III). Individual periods ranged between 12.6 and $20.0 \mathrm{~min}$. The means of maximum, nadir, and amplitude were also not different from normal, resulting in a similar relative amplitude (55.8 \pm 3.5 vs. $46.6 \pm 5.4 \% ; P>0.05)$. Fig. 6 shows the high-frequency insulin secretion oscillatory pattern in a pancreas transplant recipient compared with that of a normal subject. Data analysis exclud-

Table IV. Ultradian Insulin Secretion Pulse Characteristics with Continuous Enteral Nutrition (8-h steady state)

\begin{tabular}{|c|c|c|c|c|c|c|c|c|}
\hline Subject & Duration & Interval & Period & Maximum & Nadir & Amplitude & $\begin{array}{c}\text { Relative } \\
\text { amplitude }\end{array}$ & ISR \\
\hline & & $\min$ & & & $\mathrm{mU} / \mathrm{min}$ & & $\%$ & $U / h$ \\
\hline \multicolumn{9}{|c|}{$\begin{array}{l}\text { Pancreas transplant } \\
\text { recipients }\end{array}$} \\
\hline 1 & 80 & 10 & 90 & 33.7 & 15.9 & 17.7 & 53 & 1.46 \\
\hline 2 & 93 & 13 & 106 & 45.4 & 12.2 & 33.0 & 73 & 1.62 \\
\hline 3 & 54 & 10 & 64 & 21.7 & 11.1 & 10.6 & 49 & 0.96 \\
\hline 4 & 72 & 10 & 82 & 22.3 & 9.4 & 12.9 & 58 & 0.99 \\
\hline 5 & 88 & 10 & 98 & 26.3 & 13.3 & 13.1 & 50 & 1.13 \\
\hline 6 & 68 & 10 & 78 & 27.3 & 17.9 & 9.4 & 35 & 1.32 \\
\hline Mean \pm SEM & $75.8 \pm 5.8$ & $10.5 \pm 0.5$ & $86.3 \pm 6.1$ & $29.5 \pm 3.6$ & $13.3 \pm 1.3$ & $16.1 \pm 3.6^{*}$ & $53 \pm 5^{*}$ & $1.25 \pm 0.11$ \\
\hline \multicolumn{9}{|l|}{ Normal subjects } \\
\hline Mean \pm SEM & $81.4 \pm 4.9$ & $11.1 \pm 0.7$ & $92.5 \pm 5.1$ & $24.4 \pm 1.5$ & $16.5 \pm 1.5$ & $7.9 \pm 0.8$ & $32.6 \pm 3.3$ & $1.25 \pm 0.09$ \\
\hline
\end{tabular}

* Significantly higher than normal, $P<0.025$ and less. 

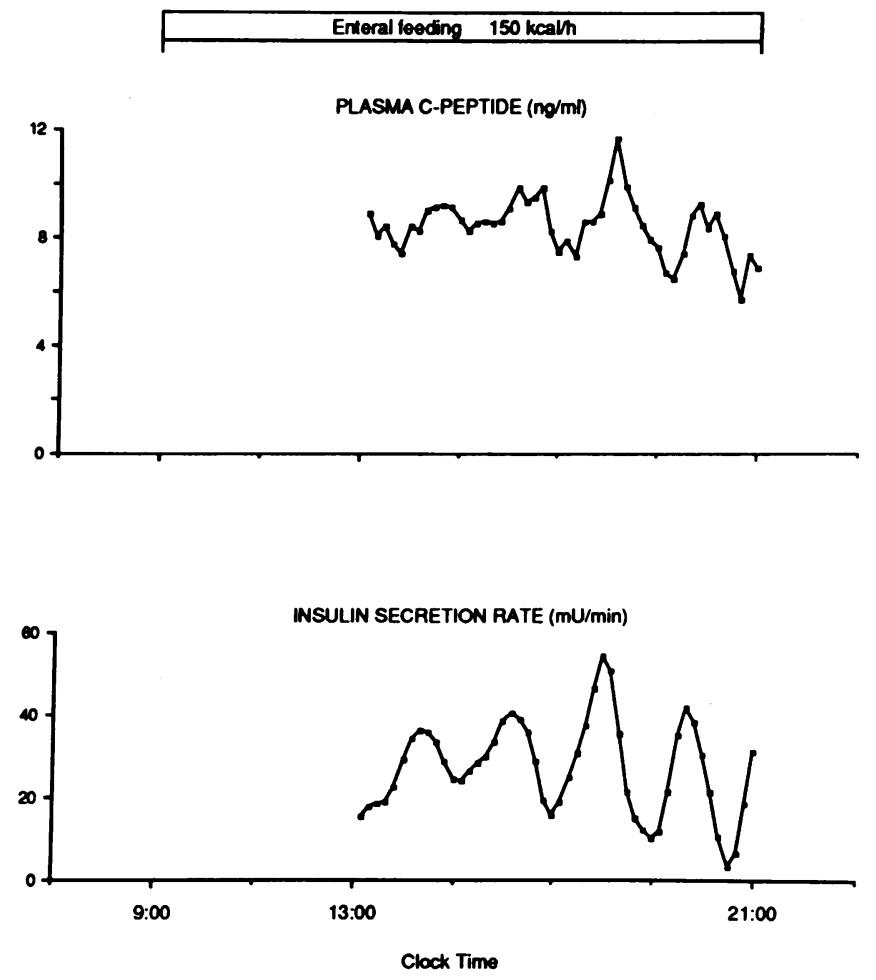

Figure 5. Low-frequency ultradian pulsatility of plasma C-peptide and insulin secretion rate during the steady state $(8 \mathrm{~h})$ of continuous enteral feeding in a pancreas transplant recipient. C-peptide concentrations were deconvoluted using the individual C-peptide coefficients to calculate the insulin secretion rate. Data were subjected to polynomial smoothing before pulse detection.

ing the two male subjects in the transplanted group did not influence these conclusions.

\section{Discussion}

In normal subjects, low-frequency ultradian insulin secretion pulses with periodicities of 75-115 min were consistently observed during the 24-h secretory cycle. Pulse periodicity and relative amplitude were similar in the overnight period and during the steady state stimulation of insulin secretion by continuous enteral feeding. High-frequency sampling confirmed the presence of high-frequency insulin secretion pulsations with a periodicity of $14-20 \mathrm{~min}$ in the fasting state. Pancreas transplant recipients demonstrated both low-frequency ultradian and high-frequency insulin secretion pulses. The high-frequency pulse characteristics were similar to normal. The lowfrequency ultradian pulse periodicity was also normal. Both the absolute and relative ultradian pulse amplitude, however, were significantly larger.

In the present study, pulsatility characteristics were evaluated after deconvoluting the pulsatile fluctuations in plasma C-peptide concentrations and calculation of insulin secretion rates $(21,22)$. This allowed evaluation of insulin delivery into the prehepatic compartment. The potential dampening effect of hepatic insulin extraction when pulsatility evaluations were performed on peripheral plasma insulin concentrations (1-6, 9) was, therefore, avoided. Continuous enteral feeding provided a more physiological stimulus than intravenous glucose
(10) while avoiding potential masking effects of the large meal-induced insulin secretory responses during interrupted feeding (8).

In normal subjects, ultradian pulse periodicity and relative amplitude were uninfluenced by the level of insulin secretion and were preserved during the 24-h secretory cycle. This consistency suggests that this mode of insulin secretion pulsatility is tightly regulated. The pancreatic low-frequency pulse periodicity was approximately similar to the ultradian rhythmicity of hypothalamically driven pituitary hormone release (29-31). This suggests that pancreatic insulin secretion might be coupled to a common central pulse generator. Ultradian secretion of pituitary hormones (e.g., luteinizing and growth hormones) when determined after deconvolution of the plasma concentration with the metabolic clearance kinetic coefficients exhibit punctuated burst-like peaks (32-34). Insulin secretion pulses on the other hand demonstrated an intervening tonic mode that amounted to $\sim 70 \%$ of the apparent maximum pulse height. The reason for this discrepancy is unknown. One possible explanation is that the ultradian insulin release profile might represent a combination of two main secretory processes representing a "pulsatile" and "tonic" mode. Alternatively, ultradian B cell insulin secretion, like pituitary hormones, occurs in punctuated burst-like pulses and the apparent tonic mode results from fusion of overlapping pulses.
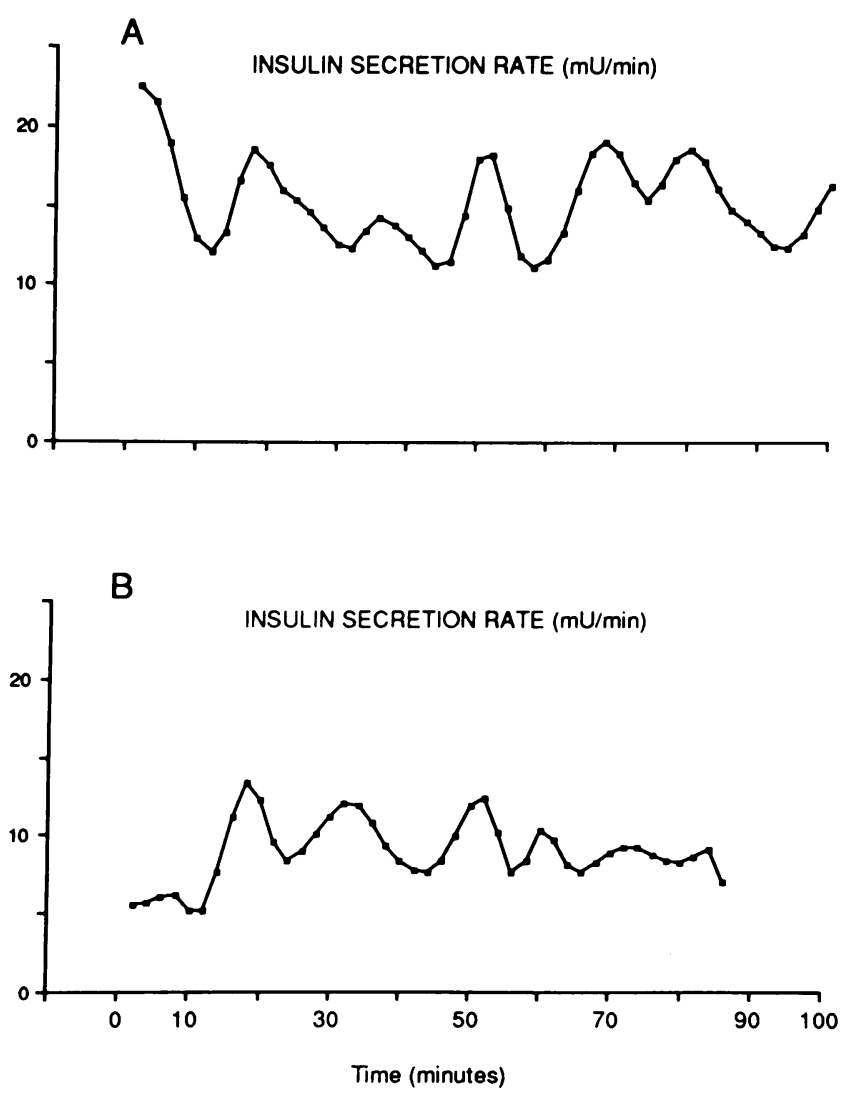

Figure 6. High-frequency pulsatility of insulin secretion rate in $(A)$ a normal subject and $(B)$ a pancreas transplant recipient. After an overnight fast, blood samples were continuously withdrawn over 2min intervals and analyzed for plasma C-peptide concentrations. Insulin secretion rates were calculated from the plasma concentrations and the kinetic coefficients of C-peptide. Data were subjected to polynomial smoothing before pulse detection. 
The high-frequency insulin secretion pulsatility showed a periodicity of $\sim 16 \mathrm{~min}$, which is slightly longer than previously reported from analysis of peripheral insulin concentration data. The demonstration of high-frequency secretory pulsations in human pancreas explants in vivo proves that their detection in the in vitro and ex vivo systems is not an artifact of the perfusion or other unphysiological settings. This high-frequency mode appears to be superimposed on the broader, lowfrequency ultradian pulses. High-frequency sampling, necessary to evaluate this pulse mode over extended periods, has limited accurate assessment of this relationship. In one subject studied by Simon et al. (9), high-frequency pulsations were superimposed on ultradian pulses. In a 2-min sampling protocol, we also observed superimposition of similar high-frequency pulsations on ultradian pulses detected during periods of fasting and enteral feeding (unpublished results).

Pancreas transplant recipients served as an in vivo model of autonomic pancreatic denervation. Subjects were metabolically stable with normal fasting and nutrient-stimulated plasma glucose and insulin secretory rate. Subjects presented with peripheral hyperinsulinemia, presumably due to drainage of secreted insulin directly into the systemic circulation. Indeed, the coexistence of normal secretion rate suggests that the hyperinsulinemia is caused by decreased metabolic clearance of the hormone. Bypassing the large hepatic extraction during the first portal passage (35) is, therefore, likely to be a factor. On the other hand, the decreased C-peptide fractional removal rate suggests that diminished renal clearance of peptides (including insulin) may also contribute to the hyperinsulinemia. Our findings are supported by the recent report by Katz and co-workers (36). They observed elevated plasma C-peptide levels in patients with kidney transplants. The degree of elevation was similar to that observed in patients who had received the combined kidney/pancreas transplants. Plasma insulin levels, however, were higher in the latter group.

Our data is the first demonstrating that in vivo occurring insulin secretion pulsatilities are generated independent of extrinsic innervation. The grafts exhibited both the high- and low-frequency pulsatility patterns. Insulin secretion high-frequency pulsations were identical to normal, excluding any significant role of the extrinsic innervation on their characteristics. These in vivo findings are consistent with those found with the isolated canine pancreas $(17,18)$ and suggest that these pulses are likely to be generated via islet or B cell activities (19). The detection of low-frequency ultradian insulin secretion pulses with normal duration, interval, and period in subjects with denervated pancreas excludes a direct role for the autonomic nervous system in the generation of this form of pulsatile release. An indirect mechanism mediated via circulating or local neurohumoral factors, however, can not be excluded. On the other hand, denervation was associated with approximately twofold larger pulse amplitudes shifting the proportion of the pulsatile mode from $\sim 30$ to $53 \%$ of the maximum pulse height. Our data, thus, raises the intriguing question whether extrinsic innervation could play a physiological role preventing extreme excursions in pulsatile hormone release and hence participate in the tight regulation of this pulsatile mode observed in normal subjects. Whereas the low-frequency ultradian secretory pulses exhibited significant increases in pulse amplitude, no significant abnormalities were observed with the high-frequency pulses. These differences suggest that the generation and possibly the regulation of these two forms of pulsatile modes are mediated by independent or distinct mechanisms.

Finally, we do recognize the fact that the pancreas transplant recipients were receiving immunosuppressive medications and the possibility that these influenced our conclusions. The most striking finding in this study, however, is the presence and not the absence of normal periodicity of both the lowand high-frequency secretory pulsatilities despite extrinsic denervation, organ procurement, and immunosuppressive medication. Furthermore, the relative pulse amplitude of the low-frequency ultradian mode was increased and not decreased. The high-frequency mode, on the other hand, was normal. It is therefore unlikely that these pulses are generated and selectively regulated by the medications received.

In conclusion, ultradian insulin release pulses represent a consistent feature of the 24-h secretory cycle in normal subjects. Pulse periodicity and relative gradient between maximum and nadir were similar in the unstimulated and nutrientstimulated states. The presence of ultradian pulses with normal periodicity in pancreas transplant recipients excludes direct role of extrinsic innervation in the pulse-generating process. The larger pulse amplitude suggests that innervation might be a necessary modulator, exerting dampening effects.

\section{Acknowledgments}

The authors are indebted to Dr. David Rodbard, Division of Computer Research and Technology, National Institutes of Health, Bethesda, MD who provided the Detect program and continued consultation and criticism. Dr. R. Mueller and the Academic Computer Services Division of the Milwaukee School of Engineering provided invaluable computer services necessary for accomplishing this work. We thank Noreen Wynn and Mark Piroli for their assistance in the analytical procedures.

This work was supported by grant HL-34989 from the National Institutes of Health and the General Clinical Research Center grant RR00058.

\section{References}

1. Goodner, C. J., B. C. Walike, D. J. Koerker, J. W. Ensinck, A. C. Brown, E. W. Chideckel, J. Palmer, and L. Kalnasy. 1977. Insulin, glucagon, and glucose exhibit synchronous sustained oscillations in fasting monkeys. Science (Wash. DC). 195:177-179.

2. Lang, D. A., D. R. Matthews, D. Peto, and R. C. Turner. 1979. Cyclic oscillations of basal plasma glucose and insulin concentrations in human beings. N. Engl. J. Med. 301:1023-1027.

3. Hansen, B. C., K. C. Jen, S. B. Pek, and R. A. Wolfe. 1982. Rapid oscillations in plasma insulin, glucagon, and glucose in obese and normal weight humans. J. Clin. Endocrinol. Metab. 54:785-792.

4. Goodner, C. J., F. G. Hom, and D. J. Koerker. 1982. Hepatic glucose production oscillates in synchrony with the islet secretory cycle in fasting rhesus monkeys. Science (Wash. DC). 215:1257-1260.

5. Lang, D. A., D. R. Matthews, M. Burnett, G. M. Ward, and R. C. Turner. 1982. Pulsatile, synchronous basal insulin and glucagon secretion in man. Diabetes. 31:22-26.

6. Hansen, B. C., K. C. Jen, S. B. Pek, and R. A. Wolfe. 1985. Fluctuations in basal plasma levels of pancreatic polypeptide in monkeys and humans. Am. J. Physiol. 248:R739-R747.

7. Jaspan, J. B., E. Lever, K. S. Polonsky, and E. Van Cauter. 1986. In vivo pulsatility of pancreatic islet peptides. Am. J. Physiol. 251:E215-E226.

8. Polonsky, K. S., B. D. Given, and E. Van Cauter. 1988. Twenty-four-hour profiles and pulsatile patterns of insulin secretion in normal and obese subjects. $J$. Clin. Invest. 81:442-448.

9. Simon, C., G. Brandenberger, and M. Follenius. 1987. Ultradian oscillations of plasma glucose, insulin, and C-peptide in man during continuous enteral nutrition. J. Clin. Endocrinol. Metab. 64:669-674.

10. Shapiro, E. T., H. Tillil, K. S. Polonsky, V.S. Fang, A. H. Rubenstein, and E. Van Cauter. 1988. Oscillations in insulin secretion during constant glucose infusion in normal man: relationship to changes in plasma glucose. J. Clin. Endocrinol. Metab. 67:307-314. 
11. Weigle, D. S. 1987. Pulsatile secretion of fuel-regulatory hormones. Diabetes. 36:764-775.

12. Matthews, D. R., B. A. Naylor, R. G. Jones, G. M. Ward, and R. C. Turner. 1983. Pulsatile insulin has greater hypoglycemic effect than continuous delivery. Diabetes. 32:617-621.

13. Bratusch-Marrain, P. R., M. Komjati, and W. K. Waldhausl. 1986. Effcacy of pulsatile versus continuous insulin administration in hepatic glucose production and glucose utilization in type I diabetic humans. Diabetes. 35:922-926.

14. Paolisso, G., S. Sgambato, R. Torella, M. Varricchio, A. Scheen, F. D'onofrio, and P. J. Lefebvre. 1988. Pulsatile insulin delivery is more efficient than continuous infusion in modulating islet cell function in normal subjects and patients with type I diabetes. J. Clin. Endocrinol. Metab. 66:122-126.

15. Goodner, C. J., I. R. Sweet, and H. Courtenay-Harrison. 1988. Rapid reduction and return of surface insulin receptors after exposure to brief pulses of insulin in perifused rat hepatocytes. Diabetes. 37:1316-1323.

16. Stagner, J. I., E. Samols, and G. C. Weir. 1980. Sustained oscillations of insulin, glucagon, and somatostatin from the isolated canine pancreas during exposure to a constant glucose concentration. J. Clin. Invest. 65:939-942.

17. Stagner, J. I., and E. Samols. 1985. Perturbation of insulin oscillations by nerve blockade in the vitro canine pancreas. Am. J. Physiol. 248:E516-E521.

18. Stagner, J. I., and E. Samols. 1985. Role of intrapancreatic ganglia in regulation of periodic insular secretion. Am. J. Physiol. 248:E522-E530.

19. Longo, E. A., K. Tornheim, J. T. Deeney, B. A. Varnum, D. Tillotson, M. Prentkill, and B. E. Corkey. 1991. Oscillations in cytosolic free $\mathrm{Ca}^{2+}$, oxygen consumption and insulin secretion in glucose stimulated rat pancreatic islets. $J$. Biol. Chem. 266:9314-9319.

20. Nghiem, D. D., and R. J. Corry. 1987. Technique of simultaneous renal pancreatoduodenal transplantation with urinary drainage of pancreatic secretion. Am. J. Surg. 153:405-406.

21. Peiris, A. N., R. A. Mueller, G. A. Smith, M. F. Struve, and A. H. Kissebah. 1986. Splanchnic insulin metabolism in obesity. Influence of body fat distribution. J. Clin. Invest. 78:1648-1657.

22. Polonsky, K. S., J. Licinio-Paixao, B. D. Given, W. Pugh, P. Rue, J. Galloway, T. Karrison, and B. Frank. 1986. Use of biosynthetic human C-peptide in the measurements of insulin secretion rates in normal volunteers and type I diabetic patients. J. Clin. Invest. 77:98-105.

23. Veldhuis, J. D., W. S. Evans, A. D. Rogol, C. R. Drake, M. O. Thorner, G. R. Merriam, and M. L. Johnson. 1984. Intensified rates of venous sampling unmask the presence of spontaneous, high-frequency pulsations of luteinizing hormone in man. J. Clin. Endocrinol. Metab. 59:96-102.
24. Rodbard, D. 1974. Statistical quality control and routine data processing for radioimmunoassays and immunoradiometric assays. Clin. Chem. 20(10):1255-1270.

25. Berman, M., and M. F. Weiss. 1978. SAAM Manual. NIH, U.S. Department of Health, Education, and Welfare. 78-180.

26. Eaton, R. P., R. C. Allen, D. S. Schade, K. M. Erickson, and J. Standefer. 1980. Prehepatic insulin production in man: kinetic analysis using peripheral connecting peptide behavior. J. Clin. Endocrinol. Metab. 51:520-528.

27. Oerter, K. E., V. Guardabasso, and D. Rodbard. 1986. Detection and characterization of peaks and estimation of instantaneous secretory rate for episodic pulsatile hormone secretion. Comput. Biomed. Res. 19:170-191.

28. Guardabasso, V., G. DeNicolao, M. Rocchetti, and D. Rodbard. 1988. Evaluation of pulse-detection algorithms by computer simulation of hormone secretion. Am. J. Physiol. 255:E775-E784.

29. Veldhuis, J. D., and M. L. Johnson. 1986. Cluster analysis: a simple, versatile and robust algorithm for endocrine pulse detection. Am. J. Physiol. 250:E486-E493.

30. Santen, R. J., and C. W. Bardin. 1973. Episodic luteinizing hormone secretion in man. Pulse analysis, clinical interpretation, and physiologic mechanisms. J. Clin. Invest. 52:2617-2628.

31. Marshall, J. C., and R. P. Kelch. 1986. Gonadotropin-releasing hormone: role of pulsatile secretion in the regulation of reproduction. N. Engl. J. Med. 315:1459-1468.

32. Veldhuis, J. D., R. J. Urban, I. Z. Beitins, R. M. Blizzard, M. L. Johnson, and M. L. Dufau. 1989. Pathophysiological features of the pulsatile secretion of biologically active luteinizing hormone in man. J. Steroid. Biochem. 33:739-749.

33. Albertsson-Wikland, K., S. Rosberg, E. Libre, L.-O. Lundberg, and T. Groth. 1989. Growth hormone secretory rates in children as estimated by deconvolution analysis of 24-h plasma concentration profiles. Am. J. Physiol. 257:E809-E814.

34. Hindmarsh, P. C., D. R. Matthews, C. Brain, P. J. Pringle, and C. G. D. Brook. 1990. The application of deconvolution analysis to elucidate the pulsatile nature of growth hormone secretion using a variable half-life of growth hormone. Clin. Endocrinol. 32:739-747.

35. Field, J. B. 1973. Extraction of insulin by the liver. Annu. Rev. Med. 24:309-314.

36. Katz, H., M. Homan, J. Velosa, P. Robertson, and R. Rizza. 1991. Effects of pancreas transplantation on postprandial glucose metabolism. N. Engl. J. Med. $325: 1278-1283$ 